\title{
Tics and Tourette Syndrome
}

\author{
1,2,3 Daryl Efron \\ ${ }^{4,5}$ Russell C Dale \\ ${ }^{1}$ Murdoch Children's Research Institute \\ ${ }^{2}$ Associate Professor, University of Melbourne Department of Paediatrics \\ ${ }^{3}$ Department of Medicine, Royal Children's Hospital, Parkville Vic 3052 \\ ${ }^{4}$ Professor of Paediatric Neurology, University of Sydney \\ ${ }^{5}$ Department of Neurology, Children's Hospital at Westmead, Westmead NSW 2145
}

Correspondence: Dr Daryl Efron, Murdoch Children's Research Institute, Flemington

Road. Parkville Vic 3052, Australia

$\mathrm{Ph}:+61-3-9345-4563$

Fax: +61-3-9345-4751

Email: daryl.efron@,rch.org.au

\section{KEY WORDS}

Tourette Syndrome, tics, obsessive-compulsive disorder

\section{ABBREVIATIONS \\ TS - Tourette Syndrome}

This is the author manuscript accepted for publication and has undergone full peer review but has not been through the copyediting, typesetting, pagination and proofreading process, which may lead to differences between this version and the Version of Record. Please cite this article as doi: $10.1111 /$ jpc.14165

This article is protected by copyright. All rights reserved. 
OCD - obsessive-compulsive disorder

ADHD - attention deficit hyperactivity disorder

ASD - autism spectrum disorder

\begin{abstract}
Tourette Syndrome (TS) is a heterogeneous disorder. The genetic basis is complex, and both in utero and ex utero environmental factors may modify the phenotypic expression of the disorder. Inflammation related to aberrations in immune activation appears to play a pathogenic role in some cases. Multiple neurochemical pathways are involved. Rather than being a pure movement problem, tics are now understood to also have a sensory component. This has resulted in new psychological therapeutic strategies and other potential treatments. Furthermore comorbidities are common, particularly ADHD, anxiety and OCD, and often cause more difficulties than the tics. The approach to treatment is dependent on the degree and types of impairment. For many patients education, acceptance and understanding are all that is needed. In more severe cases psychological and/or pharmacological interventions may be indicated. In this article the clinical features and pathophysiology of TS are reviewed, and a pragmatic management approach is discussed.
\end{abstract}

This article is protected by copyright. All rights reserved. 
Tics have been of interest to neurologists and psychiatrists for centuries. In 1885 Georges Gilles de la Tourette, encouraged by his mentor neurologist Jean-Martin Charcot, reported a series of 9 patients with "maladie des tics convulsifs avec coprolalie", distinguishing it from chorea and hysteria. In fact another famous French physician Jean Itard had first reported one of these cases in 1825, a noblewoman notorious for swearing loudly in the middle of conversations.(1) Through much of the $20^{\text {th }}$ century psychoanalytic theory prevailed, contending that tics were the result of repressed masturbatory desires. These explanatory assumptions were prominent until the 1970s when neurobiological understandings began to develop, partly in response to the observation that haloperidol can be helpful in treatment.

This article will focus on clinical assessment and management, with some background discussion of epidemiology and current understandings of aetiology and pathogenesis.

\section{Definitions}

Tourette Syndrome (TS, also called Tourette Disorder) is a neurodevelopmental disorder characterised by chronic vocal and motor tics causing distress and functional impairment. DSM-5 criteria require the presence of both vocal and motor tics over more than 12 months.(2) If there are only either vocal or motor tics then the term chronic tic disorder is used (see Box 1).

This article is protected by copyright. All rights reserved. 
[INSERT Box 1 here]

This article is protected by copyright. All rights reserved. 


\section{Epidemiology and natural history}

Transient tics occur in up to $25 \%$ of healthy children. Chronic tic disorder has a prevalence of approximately 1-3\%. Prevalence estimates for TS in childhood vary from $0.1 \%$ to $1 \%$, with a male:female ratio of approximately $4: 1 .(3,4)$.

The commonest age of onset of TS is the early primary school years, although it sometimes begins earlier. Motor tics usually precede vocal tics. Tics tend to follow an unpredictable waxing and waning pattern over time. Maximum severity is typically between 10-12 years of age, with substantial improvement by late adolescence in the majority of cases.(5) $75 \%$ of TS patients have reduced severity by adulthood, although many adults still have mild tics.(4)

\section{Tic phenomenology}

Tics are sudden movements or vocalisations, which are repetitive and stereotypical. Common motor tics include eye blinking, jerks of head, shoulders and torso, and facial grimaces; vocal tics include humming noises, throat clearing, sniffing, grunting, and squealing, and less commonly calling out a word or phrase.

Tics change over time, with one tic replaced by another. In severe cases tics can become complex involving sequences of coordinated movements, such as arm straightening, tapping, jumping, hopping, body gyrations, obscene gestures or bizarre gait. Tics occasionally become 'linked' such that one tic leads rapidly to another and 
then another, in a repertoire unique to the individual. Superimposed voluntary compensatory tic suppression behaviours often contribute to the presentation.(6)

Echophenomena (echolalia and echopraxia - mimicking of words and gestures respectively) occur in nearly half of TS patients, and are associated with comorbid obsessionality.(4) Coprolalia occurs in $15-20 \%$ of individuals with TS, with onset typically about 5 years after tic onset. $(4,7)$ Coprolalia is more common in males, and is associated with spitting, smelling of objects and inappropriate sexualised behaviour.(8)

Tics have a sensory build-up component (premonitory urge), which precedes the tic and is relieved transiently by the movement or noise. The premonitory urge is usually not recognized or able to be described by young children;(4) adolescents often describe it vaguely as like an itch, which is relieved following execution of the tic. In contrast to involuntary movement disorders such as chorea and dystonia, tics are suppressible, at the cost of the accumulation of inner tension. Another important feature is suggestibility: mentioning the tics or being asked about them can bring on a need to perform the tic.

Tics fluctuate in severity from hour-to-hour, day-to-day, and week-to-week, with a 'waxing and waning' pattern over time(4). Times where the tics are more frequent may be associated with an identifiable stress such as starting a new school year, however often there is no obvious environmental factor triggering a more severe 
period. Parents often report that tics are highly intense after the school day, e.g. in the car on the way home, possibly related to conscious or subconscious suppression at school, followed by a 'release' of the tics. Tics can also occur in sleep.

\section{Comorbidities}

Up to $85 \%$ of children with TS have one or more neurodevelopmental or mental health comorbidities, and comorbidity is associated with worse functional outcomes $(4,9)$. The most frequent comorbidities are attention deficit hyperactivity disorder (ADHD, seen in 54\% of TS patients) and obsessive-compulsive disorder (OCD, 50\%).(9) OCD in the context of tic disorders has a slightly different phenotype, with more counting, aggressive thoughts, symmetry, and touching compared to OCD without tics, which has more contamination compulsions. The coexistence of tics with OCD has generated the concept of obsessive-compulsive tic disorder, which is more likely to have earlier symptom onset, male gender, sensory phenomena and ADHD(10). Discriminating a tic from a compulsion can sometimes be challenging, but is therapeutically important. Tics typically have the 'premonitory urge', whereas compulsions are typically associated with obsessions and prominent anxiety.

Other comorbidities more commonly observed in children with TS include anxiety, externalising disorders (i.e. oppositional defiant disorder, conduct disorder), learning disorders, sleep disorders, impaired social cognition and sensory processing difficulties.(9). Autistic spectrum disorder (ASD) has been diagnosed in 5-15\% of TS cohorts(11). A recent study using the Social Responsiveness Scale found that $23 \%$ of 
TS patients met ASD criteria, although this was primarily due to higher scores in the repetitive and restricted behaviors subscale, highlighting the importance of social communication impairments in making an ASD diagnosis in a child with TS.(11) Self-injurious behavior occurs in a minority of TS patients, particularly in those who are highly obsessional and those with ASD.

\section{Pathophysiological hypotheses}

The pathophysiology of tics is not well understood, but is believed to involve abnormalities in the pathways between the cerebral cortex and basal ganglia, leading to a background neuronal disinhibition in both motor and limbic systems.(12) Aligning with the clinical association that sensory features are common, sensory limbic and executive corticostriatal loops have also been demonstrated to be affected.

$(12,13)$ Biochemically TS appears to result from alteration in both dopaminergic modulation and histaminergic transmission, $(12,13)$ although other neurotransmitter systems may also be involved.

\section{Neuroimaging findings}

Neuroimaging studies have demonstrated a range of differences in the brains of children with TS compared to healthy controls. Structural MRI findings have been somewhat inconsistent, but have included thinning of the sensorimotor and premotor cortices, reduced bilateral caudate volumes, (14) alterations in cerebellar morphology,(15) and fronto-striatal white matter changes.(16) Functional MRI studies 
have indicated that children with TS require increased activation in fronto-striatal circuitry to suppress tics during task performance.(17)

\section{Aetiology}

\section{Genetic vulnerability}

TS appears to be strongly genetic,(18) with a polygenic inheritance pattern most likely. It is between 10 and 100 times more common in first degree family members of TS patients compared with the general population(19). Environmental factors may contribute to the risk of phenotypic expression of TS.

TS genetic research methods have included large scale collaborative genome-wide association studies, copy number variation (CNV) studies and trio studies for rare large effect mutations. In addition some groups have explored gene-environment interactions with a focus on infection and immunological differences.(20, 21) Although hundreds of genes appear to be involved, only a small proportion of risk has been explained.(22) Some of the genetic vulnerability may not be solely transmitted in 'neurological or psychiatric genes' but may include immunogenetic risk factors, as is now recognized in autistic spectrum disorder. Methylation and other epigenetic factors appear to influence gene expression in TS, $(23,24)$ supporting the importance of environmental contributions to the phenotype. Recent studies also emphasize the importance of deep phenotyping in genotype correlation studies, particularly the overlapping relationships between TS and OCD (25)and ADHD.(26)

This article is protected by copyright. All rights reserved. 
In summary, the genetic inheritance in TS is complex and involves modest contribution from rare de novo mutations, some contribution of $\mathrm{CNV}$ and epigenetic factors, and overlap with ASD. $(27,28)$

\section{Immunological factors}

A small subgroup of patients present with the acute onset of florid tics in association with behavioural changes (particularly obsessive-compulsive behaviours, emotional lability), sometimes following an infection such as group A streptococcal or Mycoplasma. The PANDAS (paediatric autoimmune neuropsychiatric disorders associated with streptococcal infection) hypothesis states that streptococcal infections trigger immune responses that interact with the brain.(29) PANDAS has evolved into PANS (paediatric acute neuropsychiatric syndrome), which removes the necessity of streptococcal infection and broadens the definition to an acute and sudden onset of tics and OCD.(30) Although PANDAS and PANS remain controversial, there are undoubtedly patients who are different to 'conventional Tourette-OCD', who have a well-documented acute onset of OCD and/or tics, often associated with a sudden behavioral and personality change with sensory symptoms, anxiety and other change in function(30). These patients are sometimes investigated for encephalitis; neuroimaging and CSF studies are normal. These patients have a high rate of familial (particularly maternal) autoimmune disorders, suggesting a potential genetic vulnerability to immune dysregulation(31). There is some evidence that these patients (particularly those with severe tics) can respond to antibiotics(32), or to immune 
therapy with steroids or intravenous immunoglobulin (at least initially)(33), although more data is required to be definitive.

There is a large literature exploring immune dysregulation in TS. The findings share many similarities with evidence of immune alterations observed in ASD. $(34,35)$ These include epidemiological linkage with maternal autoimmunity, elevated cytokines in blood and/or CSF, elevated mRNA of immune genes in blood, microglial activation suggesting neuro-inflammation, and induction of symptoms by infection/inflammation in animal models.(36) Taken together, these recent studies provide evidence that infection or inflammation in utero or ex utero is associated with TS expression.(37) Other environmental factors including in utero risk factors, perinatal factors and stress have been recently reviewed.(38)

\section{Clinical assessment}

As discussed, chronic tic disorder and TS often present in the context of other developmental and mental health disorders, a patient group sometimes referred to as having "mixed developmental disorders". The tics may or may not be the most prominent or impairing feature of the presentation. In any case, careful evaluation for the common comorbidities is an essential component of the paediatric assessment.

Tics may be observed in the consulting room, or parents may have video of them on their phones or other devices. In some cases it is not entirely clear whether the symptoms are tics, stereotypy, compulsion, or a movement disorder such as chorea. 
The presence on history of a premonitory urge with relief following the movement, as well as the ability to suppress the movement, supports the diagnosis of tics.

Although minor tics usually do not affect the individual greatly, more severe tics can be extremely impairing. The impairment may be influenced by the severity of the tics, how 'camouflagable' the tics are, the effort involved in camouflage which can be extremely distracting, and how much the tics interfere with daily activities. Tics can be painful, and forceful neck tics have been reported, rarely, to cause injuries including cervical disc herniation. Sometimes children with TS experience peer victimisation and social ostracism, and consequently may have poor self-image, anger and depression. The family's response is a key factor influencing functional status.

It is sometimes useful to quantify tics and their impairment. The clinician-rated Yale Global Tic Severity Scale (Y-GTSS) is the gold standard measure of TS in children aged 6-17 years.(39) It is a semi-structured interview to document the number, frequency, intensity, complexity, and impairment associated with motor and phonic tics. Repeated measures of the Y-GTSS can help with evaluating response to treatment.

Allied health assessment can be extremely helpful, particularly neuropsychological testing (executive function, academic function, social cognition) and occupational therapy assessment (sensory profile). Clinical psychology input can be helpful for assessment and intervention for anxiety, oppositional behavior and family dynamic difficulties, which often develop in these patients over time. 


\section{Management}

Patients with relatively mild TS do not necessarily need specific intervention for their tics. Education about the condition and its natural history is important and often extremely helpful. Screening for comorbid disorders such as ADHD, OCD, mood disorders and externalizing disorders is important as these are often more impairing than the tics and may need targeted intervention.

If the tics themselves are severe and causing functional impairments then psychological and/or pharmacological intervention may be indicated. In the authors' experience only a minority of referred children require pharmacological treatment for the tics themselves.

\section{Education}

TS can be a distressing, baffling and stigmatizing condition. Education for the patient, family, and school is a key element in successful management. Children sometimes choose to present a talk to their class about TS; this can be highly empowering. The Tourette Syndrome Association of Australia provides support for families, including an informative website, local support groups, an annual conference and camps.

\section{Psychological treatment}

The non-pharmacological approach for TS with the best evidence is known as Comprehensive Behavioral Intervention for Tics (CBIT). CBIT involves training the 
individual to recognise the premonitory urge, and to generate voluntary competing responses which are incompatible with the tic (habit reversal training, HRT), and/or increase their tolerance to the premonitory urge (exposure with response prevention, ERP). CBIT has been shown to be superior to supportive psychotherapy for children aged 9-17 years with TS,(40) including those with comorbid OCD and ADHD.(41) Patients who are not taking tic-suppressing medications may experience greater benefit from CBIT than those on medication.(42) As it is difficult to access trained CBIT therapists, online delivery of CBIT study is being investigated.(42) Children younger than around 10 years of age may not have awareness of the premonitory urge or be able to understand and apply the strategies, and so may not be suitable for CBIT.(43) Furthermore the success of a behavioural management program for TS depends on the active involvement and commitment of the parents.

\section{Medication}

Although a number of medications have been shown to be superior to placebo in treating tics in children and adolescents, in practice tics are typically reduced rather than eliminated, and usually continue to follow a fluctuating course. Furthermore the medications prescribed carry quite a high risk of side-effects, some of which are serious. The choice of medication is often driven in part by the patient's comorbidity profile, with the goal of targeting multiple symptoms e.g. tics and anxiety. 
The alpha-2 adrenergic agonists clonidine or guanfacine are moderately effective in reducing tics, and are often chosen first line, particularly in the common setting of comorbid ADHD which also often responds to these medications.(44) The most frequent side effect is sedation; given at night they can be effective in treating associated sleep initiation or maintenance disorders.

If the alpha agonists are ineffective or not tolerated then the second generation antipsychotics (SGA) risperidone or aripiprazole may be considered (43). These drugs are probably the most effective in reducing tics, however side effects including sedation and weight gain and associated metabolic disturbances (e.g. dyslipidaemia, insulin resistance) are often problematic. Extra-pyramidal side effects such as akathisia and tardive dyskinesia are also occasionally observed with SGAs. The first generation anti-psychotic haloperidol is also effective in treating tics, however has a higher risk of extra-pyramidal side effects (including acute dystonias) than the SGAs so is usually reserved for severe and refractory cases. Other medication options occasionally used to directly target tics include the dopamine-depleting agent tetrabenazine,(45), topiramate,(46) and baclofen,(47) although in our experience these agents are not particularly effective and/or not well tolerated.

In patients with moderate to severe ADHD and comorbid TS methylphenidate remains the most effective treatment for ADHD, and does not usually cause a worsening of tics, although this can occur. Combined methylphenidate plus clonidine may be superior to stimulant treatment alone.(48) The selective noradrenergic 
reuptake inhibitor atomoxetine is an alternative; it has some beneficial effect on both ADHD and tics.(49)

When TS is associated with severe OCD and/or generalized anxiety disorder unresponsive to first line psychological treatment such as cognitive behaviour therapy, a selective serotonin re-uptake inhibitor may be indicated e.g. fluoxetine, sertraline or fluvoxamine. In addition to being effective for the $\mathrm{OCD} /$ anxiety there may be an indirect reduction in tic severity.

\section{Novel therapies}

Botulinum toxin injection can be useful in adolescents for severe localised tics such as 'whiplash' tics, which can result in injury(50). Deep brain stimulation (DBS) of subcortical targets has been used in adults with severe medically-refractory TS, and there is some evidence suggesting a beneficial effect.(51) Its role in paediatric TS remains uncertain, and given the expected improvements in tics between 12-20 years of age, it would seem inappropriate to consider DBS in most adolescents with TS. Finally cannabinoids may have a role in the treatment of TS in future; preliminary studies have begun in adults.

\section{Summary}

TS is a complex neurodevelopmental disorder with heterogeneous aetiology. It is usually associated with multiple comorbid and/or secondary mental health disorders. 
Although the typical natural history is of improvement and often remission over time, TS can cause severe morbidity.

Management involves addressing the symptoms causing impairment, which may involve psychological and/or pharmacological treatment. It is hoped that the emerging understanding of the biological basis of TS will lead to treatments based more on the underlying physiological mechanisms, rather than just symptom control. 


\section{MCQs}

1) Which one of the following clinical features is NOT characteristic of tics?
a) Stereotypical movements
b) Premonitory urge
c) Onset in adolescence
d) Suppressability
e) Suggestibility

2) Which ONE of the following disorders is most frequently comorbid with Tourette syndrome?
a) Intellectual disability
b) Generalised anxiety disorder
c) Autism spectrum disorder
d) Obsessive compulsive disorder
e) Depression

3) Which ONE of the following medications is most effective for tics in children and adolescents?
a) Alpha agonists
b) Stimulants
c) Selective serotonin reuptake inhibitors
d) Anti-epileptic drugs
e) Baclofen

\section{Answers to MCQs}

1) c. The commonest age of onset is early primary school, sometimes earlier.

2) d. ADHD is about as common a co-morbidity as OCD (but was not an option in the MCQ). 
3) a. Alpha agonists are moderately effective but behavioural therapy is preferred.

This article is protected by copyright. All rights reserved. 


\section{References}

1. Shapiro A.K. SES, Bruun R.D., Sweet R.D. Gilles de la Tourette Syndrome. New York, NY: Raven Press; 1978.

2. American Psychiatric Association. Diagnostic and Statistical Manual of Mental Disorders, 5th edition. Arlington, VA. American Psychiatric Publishing. 2013. 3. Knight T, Steeves T, Day L, Lowerison M, Jette N, Pringsheim T. Prevalence of tic disorders: a systematic review and meta-analysis. Pediatr. Neurol. 2012;47(2):77-90.

4. Robertson MM. A personal 35 year perspective on Gilles de la Tourette syndrome: prevalence, phenomenology, comorbidities, and coexistent psychopathologies. Lancet Psychiatry. 2015;2(1):68-87.

5. Cohen SC, Leckman JF, Bloch MH. Clinical assessment of Tourette syndrome and tic disorders. Neurosci. Biobehav. Rev. 2013;37(6):997-1007.

6. Plessen KJ, Royal, J.M., Peterson, B.S. Neuroimaging of tic disorders with coexisting attention deficit hyperactivity disorder. Eur. Child Adolesc. Psychiatry 2007;16(Suppl 1):i60-i70.

7. Eapen V, Robertson MM. Are there distinct subtypes in Tourette syndrome? Pure-Tourette syndrome versus Tourette syndrome-plus, and simple versus complex tics. Neuropsychiatr. Dis. Treat. 2015;11:1431-6.

8. Freeman RD, Zinner SH, Muller-Vahl KR, et al. Coprophenomena in Tourette syndrome. Dev. Med. Child Neurol. 2009;51(3):218-27.

9. Hirschtritt ME, Lee PC, Pauls DL, et al. Lifetime prevalence, age of risk, and genetic relationships of comorbid psychiatric disorders in Tourette syndrome. JAMA Psychiatry. 2015;72(4):325-33.

10. Dell'Osso B, Marazziti D, Albert U, et al. Parsing the phenotype of obsessivecompulsive tic disorder (OCTD): a multidisciplinary consensus. Int. J. Psychiatry Clin. Pract. 2017;21(2):156-9.

11. Darrow SM, Grados M, Sandor P, et al. Autism Spectrum Symptoms in a Tourette's Disorder Sample. J.Am. Acad. Child Adolesc Psychiatry. 2017;56(7):610-7 e1.

12. Ganos C. Tics and Tourette's: Update on pathophysiology and tic control. Curr Opin Neurol. 2016;29(4):513-8.

13. Yael D, Vinner E, Bar-Gad I. Pathophysiology of tic disorders. Mov Disord. 2015;30(9):1171-8.

14. Peterson BS, Thomas $\mathrm{P}, \mathrm{Kane} \mathrm{MJ}$, et al. Basal Ganglia volumes in patients with Gilles de la Tourette syndrome. Arch Gen Psychiatry. 2003;60(4):415-24.

15. Tobe RH, Bansal R, Xu D, et al. Cerebellar morphology in Tourette syndrome and obsessive-compulsive disorder. Ann Neurol. 2010;67(4):479-87.

16. Worbe Y, Gerardin E., Hartmann A., et al. Distinct structural changes underpin clinical phenotypes in patients with Gilles de la Tourette syndrome persisting into adulthood. Brain. 2010;133:3649-60. 
17. Marsh R, Maia TV, Peterson BS. Functional Disturbances Within Frontostriatal Circuits Across Multiple Childhood Psychopathologies. Am J Psychiat. 2009;166(6):664-74.

18. Leckman JF, Bloch M. Tic disorders. In: Rutter MJ, Bishop D, Pine D et al, editors. Rutter's Child and Adolescent Psychiatry, 5th Edition. Oxford, UK.: Blackwell Publishing; 2008: 719-36.

19. Pauls DL, Fernandez TV, Mathews CA, State MW, Scharf JM. The Inheritance of Tourette Disorder: A review. J. Obsess. Compuls. Relat. Disord. 2014;3(4):380-5.

20. Georgitsi M, Willsey AJ, Mathews CA, State M, Scharf JM, Paschou P. The Genetic Etiology of Tourette Syndrome: Large-Scale Collaborative Efforts on the Precipice of Discovery. Front. Neurosci. 2016;10:351.

21. Forde NJ, Kanaan AS, Widomska J, et al. TS-EUROTRAIN: A EuropeanWide Investigation and Training Network on the Etiology and Pathophysiology of Gilles de la Tourette Syndrome. Front. Neurosci. 2016;10:384.

22. Willsey AJ, Fernandez TV, Yu D, et al. De Novo Coding Variants Are Strongly Associated with Tourette Disorder. Neuron. 2017;94(3):486-99 e9.

23. Pagliaroli L, Veto B, Aranyi T, Barta C. From Genetics to Epigenetics: New Perspectives in Tourette Syndrome Research. Front. Neurosci. 2016;10:277.

24. Zilhao NR, Padmanabhuni SS, Pagliaroli L, et al. Epigenome-Wide Association Study of Tic Disorders. Twin Res. Hum .Genet. 2015;18(6):699-709.

25. Darrow SM, Hirschtritt ME, Davis LK, et al. Identification of Two Heritable Cross-Disorder Endophenotypes for Tourette Syndrome. Am. J. Psychiatry. 2017;174(4):387-96.

26. Hirschtritt ME, Darrow SM, Illmann C, et al. Social disinhibition is a heritable subphenotype of tics in Tourette syndrome. Neurology. 2016;87(5):497-504.

27. Fernandez TV, Sanders SJ, Yurkiewicz IR, et al. Rare copy number variants in tourette syndrome disrupt genes in histaminergic pathways and overlap with autism. Biol. Psychiatry. 2012;71(5):392-402.

28. McGrath LM, Yu D, Marshall C, et al. Copy number variation in obsessivecompulsive disorder and tourette syndrome: a cross-disorder study. J. Am. Acad.

Child Adolesc. Psychiatry. 2014;53(8):910-9.

29. Swedo SE, Leonard HL, Garvey M, et al. Pediatric autoimmune neuropsychiatric disorders associated with streptococcal infections: clinical description of the first 50 cases. Am. J. Psychiatry. 1998;155(2):264-71.

30. Chang K, Frankovich J, Cooperstock M, et al. Clinical evaluation of youth with pediatric acute-onset neuropsychiatric syndrome (PANS): recommendations from the 2013 PANS Consensus Conference. J. Child Adolesc. Psychopharmacol. 2015;25(1):3-13.

31. Murphy TK, Storch EA, Turner A, Reid JM, Tan J, Lewin AB. Maternal history of autoimmune disease in children presenting with tics and/or obsessivecompulsive disorder. J. Neuroimmunol. 2010;229(1-2):243-7.

32. Murphy TK, Brennan EM, Johnco C, et al. A Double-Blind Randomized Placebo-Controlled Pilot Study of Azithromycin in Youth with Acute-Onset 
Obsessive-Compulsive Disorder. J. Child Adolesc. Psychopharmacol. 2017;27(7):640-651

33. Perlmutter SJ, Leitman SF, Garvey MA, et al. Therapeutic plasma exchange and intravenous immunoglobulin for obsessive-compulsive disorder and tic disorders in childhood. Lancet. 1999;354(9185):1153-8.

34. Rossignol DA, Frye RE. A review of research trends in physiological abnormalities in autism spectrum disorders: immune dysregulation, inflammation, oxidative stress, mitochondrial dysfunction and environmental toxicant exposures. Mol. Psychiatry. 2012;17(4):389-401.

35. Dale RC. Tics and Tourette: a clinical, pathophysiological and etiological review. Curr. Opin. Pediatr. 2017;29(6):665-73.

36. Martino D, Zis P, Buttiglione M. The role of immune mechanisms in Tourette syndrome. Brain Res. 2015;1617:126-43.

37. Estes ML, McAllister AK. Maternal immune activation: Implications for neuropsychiatric disorders. Science. 2016;353(6301):772-7.

38. Hoekstra PJ, Dietrich A, Edwards MJ, Elamin I, Martino D. Environmental factors in Tourette syndrome. Neurosci.Biobehav. Rev. 2013;37(6):1040-9.

39. Leckman JF, Riddle MA, Hardin MT, et al. The Yale Global Tic Severity Scale: initial testing of a clinician-rated scale of tic severity. J. Am. Acad. Child Adolesc. Psychiatry. 1989;28(4):566-73.

40. Piacentini J, Woods DW, Scahill L, et al. Behavior therapy for children with Tourette disorder: a randomized controlled trial. JAMA. 2010;303(19):1929-37.

41. Sukhodolsky DG, Woods DW, Piacentini J, et al. Moderators and predictors of response to behavior therapy for tics in Tourette syndrome. Neurology.

2017;88(11):1029-36.

42. Jakubovski E, Reichert C, Karch A, Buddensiek N, Breuer D, Muller-Vahl K. The ONLINE-TICS Study Protocol: A Randomized Observer-Blind Clinical Trial to Demonstrate the Efficacy and Safety of Internet-Delivered Behavioral Treatment for Adults with Chronic Tic Disorders. Front. Psychiatry. 2016;7:119.

43. Ganos C, Martino D, Pringsheim T. Tics in the Pediatric Population: Pragmatic Management. Mov. Disord Clin. Pract. 2017;4(2):160-72.

44. Weisman H, Qureshi IA, Leckman JF, Scahill L, Bloch MH. Systematic review: pharmacological treatment of tic disorders--efficacy of antipsychotic and alpha-2 adrenergic agonist agents. Neurosci.Biobehav. Rev. 2013;37(6):1162-71. 45. Porta M, Sassi M, Cavallazzi M, Fornari M, Brambilla A, Servello D. Tourette's syndrome and role of tetrabenazine: review and personal experience. Clin Drug Investig. 2008;28(7):443-59.

46. Jankovic J, Jimenez-Shahed J, Brown LW. A randomised, double-blind, placebo-controlled study of topiramate in the treatment of Tourette syndrome. $J$ Neurol. Neurosurg. Psychiatry. 2010;81(1):70-3.

47. Singer HS, Wendlandt J, Krieger M, Giuliano J. Baclofen treatment in Tourette syndrome: a double-blind, placebo-controlled, crossover trial. Neurology. 2001;56(5):599-604. 
48. Tourette's Syndrome Study Group. Treatment of ADHD in children with tics: a randomized controlled trial. Neurology. 2002;58(4):527-36.

49. Allen AJ, Kurlan RM, Gilbert DL, et al. Atomoxetine treatment in children and adolescents with ADHD and comorbid tic disorders. Neurology.

2005;65(12):1941-9.

50. Marras C, Andrews D, Sime E, Lang AE. Botulinum toxin for simple motor tics: a randomized, double-blind, controlled clinical trial. Neurology. 2001;56(5):60510 .

51. Baldermann JC, Schuller T, Huys D, et al. Deep Brain Stimulation for Tourette-Syndrome: A Systematic Review and Meta-Analysis. Brain Stimul. 2016;9(2):296-304. 


\section{Box 1. DSM-5 criteria for tic disorders (2)}

1. Tourette syndrome: Two or more motor tics and at least one vocal tic occurring many times a day nearly every day for longer than a year, with onset $<18$ years of age.

2. Persistent (chronic) motor or vocal tic disorder: One or more motor tics or vocal tics occurring many times a day nearly every day for longer than a year, with onset $<18$ years of age.

3. Provisional tic disorder: One or more motor tics or vocal tics for less than 12 months, with onset $<18$ years. 


\section{University Library}

\section{- M M I N E R VA A gateway to Melbourne's research publications}

Minerva Access is the Institutional Repository of The University of Melbourne

Author/s:

Efron, D;Dale, RC

Title:

Tics and Tourette syndrome

Date:

2018-10-01

Citation:

Efron, D. \& Dale, R. C. (2018). Tics and Tourette syndrome. JOURNAL OF PAEDIATRICS AND CHILD HEALTH, 54 (10), pp.1148-1153. https://doi.org/10.1111/jpc.14165.

Persistent Link:

http://hdl.handle.net/11343/284621 